\title{
Initial Electron Distributions for Free-Electron Lasers Generated by
Injector and Accelerator Simulations Free-Electron Lasers Generated by
Injector and Accelerator Simulations

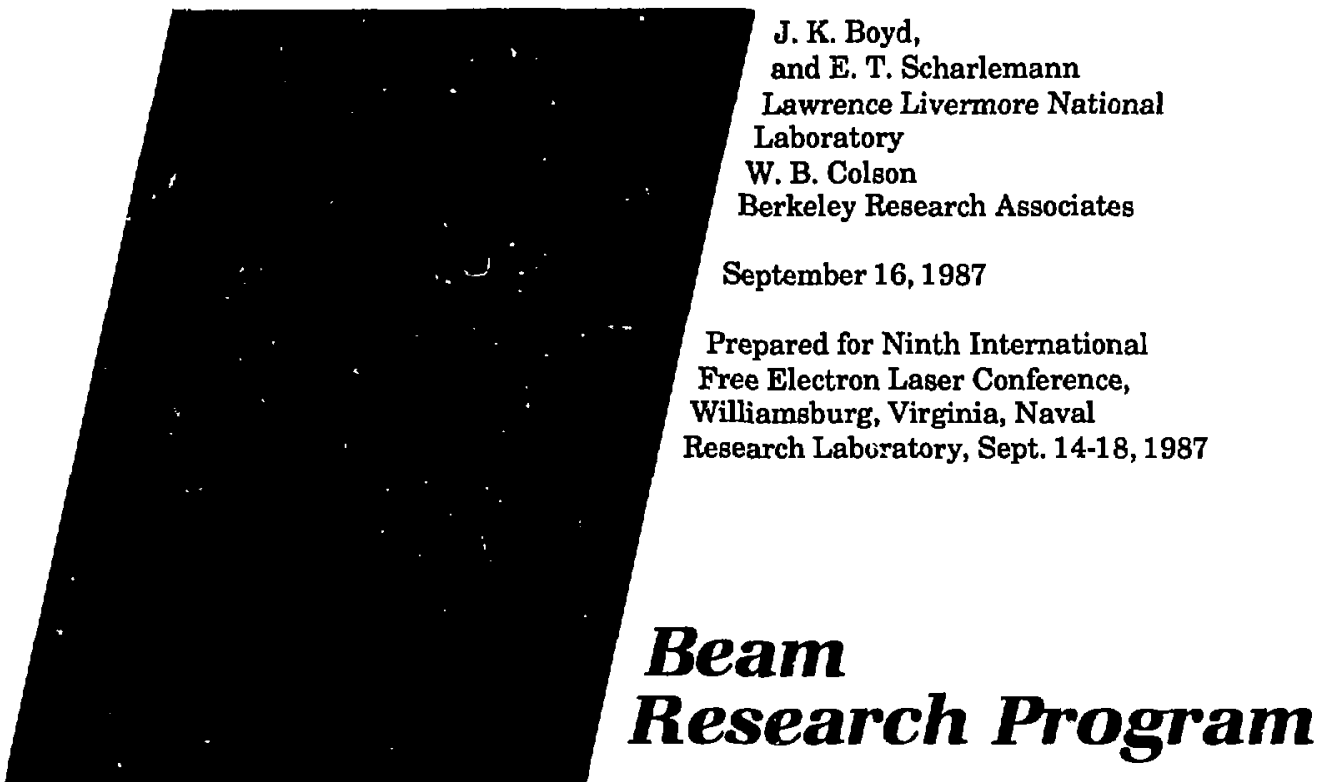

Lawrence Livermore National Laboratory 
This document uas prepared at an mccount of wow sponwored by an arency of the Unitud Staten Government. Neither the United States Gevernment wir the Unirersity of Callfornie mar any of their empleyeth makes any narranty, express or Implied, of anumes any lekal liability or respensibility for the arcuracy, completeness, or uxelulness of anj Information. apperntws, product, of precess dicloned, or reprenents that

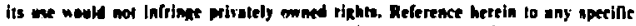

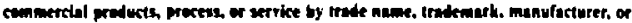

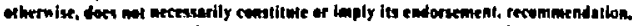
or farerine by the Lailed Siatr Government or the Universily of Califoraia. The

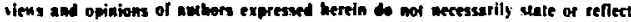

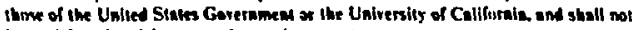
be und for morrtisina or preduct endossemest purposes. 


\title{
INITIAL ELECTRON DISTRIBUTIONS FOR FREE-ELECTRON LASERS GENERATED BY INJECTOR AND ACCELERATOR SIMULATIONS *
}

\author{
J.K.Boyd, W.B. Colson, ${ }^{\dagger}$ and E.T. Scharlemann \\ Lawrence Livermore National Laboratory, University of California \\ P.O.Box 808, L-626, Livermore, CA 94550
}

September 14, 1087

\begin{abstract}
Early free-electron laser (FEL) development was guided by simple performance criteria based on the number of undulator periods, electron beam quality, and current. The beam quality (emittance and energy spread) was used to characterize the initial distribution of axial electron velocities along the undulator axis. While the emittance and energy spread determine the overall width of the distribution, its detailed shape is also important. As new accelerators are designed specifically for FEL applications, it becomes important to obtain distribution shape information from simulations that include the electron gun, accelerator, and beam transport in addition to the usual electron/optical interaction in the undulator.

The distribution at the entrance to the undulator can be calculated from numerical simulations of the cathode emission, accelention, and transport of an electron beam. We have modeled the beam generation, from cathode emission up to the energy of the accelerator injector, using an axisymmetric, cylindrical geometry particie simulation (DPC). This code solves the relativistic force equation with fields obtained from Maxwell's equations in the Darwin model. The DPC calculation is run repeatedly varying parameters such as accelerating stress, electrode configuration, and axial magnetic field profile until a good match is obtained for the accelerator. The beam exiting from the injector can be accelerated and transported using the transfer matrix technique with a simple model for accelerating gaps and magnets. Alternatively, acceleration and transport can be simulated
\end{abstract}

\footnotetext{
* Work performed jointly under the suspices of the US DOE by LLNL under contract No. W-7405-ENG-48 and for the DOD under SDIO/SDC-ATC MIPR No. W31RPD-7-D4041

† Berkeley Research Associates, P.O.Box 241, Berkeley CA 94701
} 
with a particle code that solves for the axisymmetric evolution of a slice of an electron beam including possible emittance growth.

The phase space obtained from the accelerator can be evaluated for performance using either the simple FEL integral equation method or the more complete FRED simulation code.

\section{Introduction}

A complete free electron laser (FEL) consists of an electron injector, accelerator, and beam transport to an undulator. The FEL gain and output power are limited by the amount of electron beam current provided by the accelerator, but the fiaction of the beam energy that can ultimately be converted to optical power depends crucially on the electron distribution as determined by emittance and energy spread. It was necessary for the first FELs to consider the urdulator as an "add-on" to an existing electron source facility, but now accelerators are designed specifically for FEL application. In this paper, we model all four FEL cuinponents to provide a means of assessing the overall performance, and to explore strategies for optimization.

The earliest evaluations of FEL performance relied on simple criteria, such as number of undulator periods, to assess the effect of electron beam quality. Even though the first experiments used a high-quality electron beam, it was realized [1] that different aspects of electron beam quality could be approximately characterized by a single parameter, the longitudinal energy spread $\Delta \gamma_{\|} m c^{2}$ where $\gamma_{\|} \equiv\left(1-\beta_{\|}^{2}\right)^{-1 / 2}$, and $c \beta_{\|}$is an electron's longitudinal velocity component. Later analyses $[2,3]$ and experiments used electron beams that were less than ideal, so that it became necessary to evaluate the detrimental effects of energy spread and emittance more accurately.

In section 2, the models used to simulate the injector and accelerator are described. An example of a far-infrared ( $21 \mu \mathrm{m}$ wavelength) FEL is presented. The injector and accelerator in this example provide a distribution which can be modeled with numerical simulations (FRED), or with the FEL integral equation method described in section 3 . An alternative, idealized distribution is then compared with this first result to emphasize the different performance possible from two distributions of comparable effective energy ipread. 
This comparison highlights the clependence of FEL performance on electron distribution shape.

\section{Injector and Accelerator Simulations}

DPC injector simulation

The injector is modeled with the axisymmetric $(r, z)$ DPC (Darwin Particle Code) computer code [4]. DPC solves for beam dynamics over a region which includes cathode emission and acceleration up to the full energy of the injector. The time history of the electrons is evolved from the space-charge-limited emission which is nonrelativistic to the full injector energy where typically relativistic effects are dominant. DPC provides a numerical model where early phase space influences such as anode-cathode (A-K) gap accelerating stress, electrode configuration (diode, triode etc.) and axial magnetic profile can be evaluated. DPC has the capability of external magnetic field coils, "stair case" shaping of electrodes, and finite electrode voltage rise times. DPC solves the relativistic force equation in three dimensional cartesian $x, y, z$ coordinates

$$
m \frac{d \overrightarrow{\mathrm{u}}}{d t}=\frac{q}{c} \overrightarrow{\mathrm{E}}+\frac{q}{c \gamma} \overrightarrow{\mathrm{u}} \times \overrightarrow{\mathrm{B}}
$$

where $m$ is particle mass, $\gamma=\left(1-(v / c)^{2}\right)^{-1 / 2}, v$ is velocity, $g$ is charge, $c$ is the speed of light, $\vec{u}=\gamma \vec{v} / c, \vec{E}$ is the electric field and $\vec{B}$ is the magnetic field. Since fields are only functions of $r$ and $z$, the current and charge density are obtained from the particles by spreading these quantities in the azimuthal angle. The fields are obtained from Maxwell's equations in the Darwin limit [5]. This amounts to neglecting the solenoidal part of the displacement current in Ampere's law.

After a phase space is generated by the DPC code there are two methods which can be used to transport the beam further. Each of these methods has several assumptions which reduce the amount of computation for transport over meters of beamline. In principle DPC could be used to do the entire job, however because the mesh is uniform, a problem generally becomes unwieldy beyond a distance of $50 \mathrm{~cm}$. Consequently DPC generates a phase space which is then used as an input to either the transfer matrix method or the ST simulation code. 


\section{Transfer matrix method}

For our application the transfer matrix method [6] essentially determines the trajectory of a particle traversing a region of uniform field or a field free gap of known voltage.

$$
\left(\begin{array}{c}
x \\
y \\
u_{x} \\
u_{y}
\end{array}\right)=\left.\cdots\left[M_{g a p}\right] \cdots\left[M_{\text {olen oid }}\right] \cdots\left(\begin{array}{c}
x \\
y \\
u_{x} \\
u_{y}
\end{array}\right)\right|_{0}
$$

As shown in Eq.(2) the position and velocity at a location along the benmline is obtnined from the initial value by repeated matrix multiplication. An accelerator is modeled as a number of regions of focus in a solenoid magnet, or acceleration in a gap. More elaborate matrix representations of components, including nonuniform fields, bends or quadrupole focusing elements are available but were not needed for this work. Each particle is stepped along the beamline by matrix multiplication to advance the entire phase space. The advantage of the transfer matrix method is that a single matrix multiply can move a particle a large distance along the beamline. The disadvantage is that the self-consistent forces can only be treated approximately. This means it is best to transport the phase space up to an energy where these effects are small before the transfer matrix method is applied. The self beam space charge force has a $\gamma^{-2}$ dependence so generally for $\gamma>7$ the transier matrix metlod is applicable.

\section{ST accelerator transport simulation}

A DPC phase space can be transported more realistically than the transfer matrix method using the ST (Slice Transport) computer code. Although the ST code is more realistic it is much more computationally intensive than the transfer matrix method. In some problems it can use several orders of magnitude more computer time than the transfer matrix method. The particles which exit the DPC problem are saved as an initial phase space sor ST which then continues their orbit down the beamline. The ST code transports a slice of the beam at a fixed distance from the beam head. In contrast to DPC the ST code assumes longitudinal decoupling of particle dynamics, which implies $\partial / \partial z<<0$. It is then only necessary to solve for motion in the transverse direction. The transverse equation of motion solves for $u_{x}, u_{y}$ and the particle energy is used to obtrin $u_{z}$,

$$
\begin{gathered}
u_{z}=\left(\gamma^{2}-1-u_{x}^{2}-u_{y}^{2}\right)^{1 / 2} \\
-4-
\end{gathered}
$$


where $\gamma=1-q \phi / m c^{2}$ is given by the potential $\phi(\vec{E}=-\nabla \phi)$. The ST electric field is obtained from $E_{r}=-d \phi / d r$ with,

$$
\frac{1}{r} \frac{d}{d r} r \frac{d \phi}{d r}=-4 \pi \rho+\lambda
$$

In Eq.(4) $\lambda$ is a source modification accounting for longitudinal charge varjation which satisfies,

$$
\frac{1}{r} \frac{d}{d r} r \frac{d \lambda}{d r}=4 \pi \frac{d^{2} \rho}{d z^{2}}
$$

From $\nabla \cdot \overrightarrow{\mathrm{E}}=4 \pi \rho$ it can be seen $\lambda$ is an approximation for $\partial \mathrm{E}_{z} / \partial z$.

The ST magnetic field consists of the sum of external prescribed fields and the beam self-magnetic field. The theta component, $B_{\theta}$ is obtained by integrating the 2 component of Ampere's law,

$$
\mathrm{B}_{\theta}=\frac{4 \pi}{r c} \int_{0}^{r} J_{z} r d r
$$

The $\mathrm{z}$ component of the self-magnetic field is obtained by integrating the steady-state theta component of Ampere's law.

$$
B_{z}=\left.B_{z}\right|_{r=0}-\frac{4 \pi}{c} \int_{0}^{r} J_{\theta} d r
$$

The axis value, $\left.\mathrm{B}_{z}\right|_{\mathrm{r}=0}$ is determined by requiring zero longitudinal magnetic flux at the radial wall position.

In $\mathrm{ST}$, the longitudinal electric field $\mathrm{E}_{z}$ is neglected; however, acceleration due to gaps is modeled by increasing the potential at the wall and solving a Laplace equation for $\phi_{g a p}(r, z)$. The total $\phi$ is then $\phi_{g a p}(r, z)$ plus the solution of $\mathrm{Eq}_{\text {. }}(4)$ with a zero boundary condition. The change in $\phi$ causes $\gamma$ to increase since $\gamma \sim \phi$. There is also a radial dependence to $\gamma$ since $\phi_{\text {gap }}$ depends on $\mathrm{r}$.

Simulation example relevant to an infrared FEL

An initial electron distribution appropriate for an infrared FEL has been generated from a DPC diode simulation and subsequent transport with ST. The parameters for this problem are given in Table 1. A model accelerator generates a $2.1 \mathrm{kA}$ beam at an energy of $20 \mathrm{MV}$. The DPC injector problem region is $36 \mathrm{~cm}$ in longitudinal extent with an $8 \mathrm{~cm}$ 


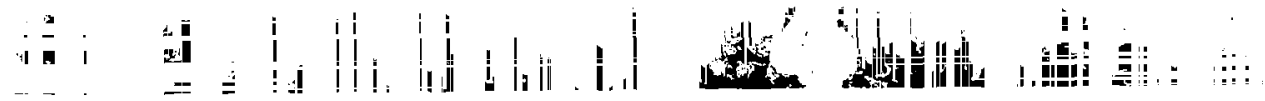

diameter cathode. The geometry, outer beam edge, and potential contours with $267 \mathrm{kV}$ spacing are plotted in Fig. 1. The emitting surface of the cathode has a $16.25 \mathrm{~cm}$ spherical radius so electrons launch cff with convergent velocities. This feature reduces the magnetic field required to catch the beam compared to ernission from a flat emitter. The A-K gap is $17.5 \mathrm{~cm}$ and the external magnetic field increases from zero to 150 gauss at $30 \mathrm{~cm}$. The magnetic field starts at zero on the calhode so there is no canonical angular momcntum added to the beam. The A-K gap voltage is $4 \mathrm{MV}$ and thus the beam is relativistic when it enters the anode. Approximately $16 \mathrm{~cm}$ into the arode the phnse space is saved to be used as an initial condition for the ST code.

The computer run time for transport in this example has been artificially reduced by assuming a rather large accelerating gradient of $4.4 \mathrm{MV} / \mathrm{m}$. Thus the $20 \mathrm{MV}$ total energy is achieved after only a distance of about $4.5 \mathrm{~m}$. Because this is a relatively short distance it was not necessary to resort to the transfer matrix method. The subsequent ST transport had 8 gaps of $2 M V$ each and a run time of 5 minutes on a Cray computer. The rms radius and magnetic field profile for this example are plotted in Fig. 2. The rms radius decreases initially, consistent with the convergent launch condition. At about $80 \mathrm{~cm}$ (as the rms radius begins to increase) the magnetic field strength is raised to 1000 gauss to hold the radius near $0.6 \mathrm{~cm}$. Again at $300 \mathrm{~cm}$ the magnetic field is increased to 900 gauss to cause minor convergence and result in a near-waist condition at $536 \mathrm{~cm}$ after the beam moves into a field free region at the end of the problem.

\section{FEL Performance Evaluation}

The phase space distribution created by the DPC/ST numerical simulation can be evaluated using either the FEL integral equation method or the FRED simulation code. Both of these methods permit a study of the field amplitude in the undulator as determined by an initial electron distribution, $f\left(\gamma_{\| l}\right)$. The DPC/ST numerical simulation we chose to work with has a $f\left(\gamma_{\|}\right)$which closely approximates a function that is linear in $\gamma_{\| 1}$ with a positive slope. The distinctive difference between the DPC/ST numerical simulation and another phase space with the same width is illustrated by comparing with an alternative idealized distribution having uniform phase space fill, $f_{u}\left(x, y, x^{\prime}, y^{\prime}\right)=$ constant. The 
$f\left(\gamma_{\| 1}\right)$ distribution corresponding to $f_{u}$ can be derived analytically to show $f\left(\gamma_{\| 1}\right)$ is linear in $\gamma_{\| 1}$ with negative slope. By adjusting parameters $f\left(\gamma_{\| 1}\right)$ for uniform phase space fill can be made to have the same width as the DPC/ST numerical simulation result. Thus, if width was the only distinguishing feature of the two distributions, each would be expected to have the same behavior. As mentioned earlier the shape of $f\left(\gamma_{11}\right)$ is important and width is not sufficient to characterize the distribution.

\section{EEL Intecral equation method}

The FEL integral equation method [7] includes an arbitrary electron distribution function in a self-consistent integral equation for the complex optical field. The integral equation for the field amplitude is derived by expanding the electron Lorentz and optical wave equations $[8]$ to first order in $a(\tau)$ (weak field) and then combining to obtain,

$$
a(r)=a_{0}+\frac{1}{2} i j \int_{0}^{\tau} d s \int_{0}^{o} d q<e^{-i \nu_{i}(s-q)}>(s-q) a(q)
$$

where $\tau=c t / L$ is the dimensionless time, $L$ is the undulator length, $\zeta=\left(k+k_{0}\right) z-\omega t$ is

the phase, $\nu=d \zeta / d r=L\left(k_{0} \beta_{z}-k\left(1-\beta_{z}\right)\right)$ is the phase velocity, $j=N\left(\frac{B \lambda_{0}}{1204 \gamma}\right)^{2} L^{2} J / I_{A}$ is the dimensionless current density, $I_{A}$ is the Alfven current, and $a=\left(\frac{17.2 \lambda_{0} B N L}{c \gamma^{2}}\right) E e^{i \varphi}$ is the dimensionless field amplitude. The initial distribution enters this formulation in the distribution average operator $\langle\ldots\rangle=\int_{-\infty}^{\infty} d \nu_{i}(\ldots) f\left(\nu_{i}\right)$. The distribution model for the DPC/ST numerical simulation is, $f_{+}\left(\nu_{i}\right)=\left(2 \sigma-\nu_{i}-\nu_{0}\right) / 2 \sigma^{2}$ and for the uniformly filled phase space with undulator focusing, $f_{-}\left(\nu_{i}\right)=\left(2 \sigma-\nu_{i}+\nu_{0}\right) / 2 \sigma^{2}$. In Fig. 3 the gain is plotted for each distribution versus width, $\sigma$ for $j=1000$. At $\sigma=100$ corresponding to the DPC/ST numerical simulation $\ln (1+G)$ is 3.1 and 0.2 for $f_{-}$and $f_{+}$respectively.

\section{FRED simulation}

The FRED code [9] solves the standard FEL equations [8] including modifications for off-axis effects in the particle dynamics and field equations. FRED uses the particle phase space generated by the DPC/ST numerical simulation so that no modeling of the distribution is necessary. The FRED code has been used to simulate the same two examples that were evaluated using the FEL integral equation method. The distribution histograms of $f\left(\gamma_{11}\right)$ for each example are plotted in Fig. 4. The uniformly filled phase space example in 
Fig. 4a approximates the predicted linear behavior with negative slope. This corresponds to $f_{-}$used with the integral equation method. The $f\left(\gamma_{\| 1}\right)$ from the DPC/ST numerical simulation is plotted in Fig. 4b. This distribution was previously modeled by $f_{+}$in the integral equation method. The gain as a function of distance along the :ndulator is plotted in Fig. 5 for the two examples run by the FRED codc. After $2 \mathrm{~m}$ the initialization with the DPC/ST numerical simulation yields a factor of 6 more gain than the uniformly filled example. This result is the opposite of the conclusion from the FEL integral equartion method. The reversal of the relative gain of $f_{-}$and $f_{+}$can be aiscribed to current profile effects-included in FRED but not in the integral equation method-that necessarily accompany the difference in $f\left(\gamma_{11}\right)$.

\section{Summary}

When beam emittance is important and the amplitude of the radiation field is small enough that it does not dominate the distribution, FEL performance is determined by the transport generated electron distribution. The numerical models used to simulate injectors and transport have been described and an example relevant to an infrared FEL has been presented. The electron distribution corresponding to this example and an alternate distribution with the same width have been analyzed using the simple FEL in'egral equation method and the more complete FRED simulation. Both methods demonstrate the dependence of FEL performance on electron distribution shape.

\section{References}

[1] J.M. Madey, J. Appl. Phys., 42 (1971) 1906.

[2] N.M.Kroll and W.A. McMullin, Pliys. Rev. A 17 (1978) 300.

[3] V.K.Neil, SRI Rep. JSR-79-10.

[4] J.K. Boyd, G.J. Caporaso, A.G. Cole, IEEE Trans. Nuc. Sci. NS-32, 5 (1985) 2602.

[5] C. W. Neilson, H. R. Lewis, Methods in Computational Physics Vol. 16 (Academic Press, N.Y., 1976) p. 367.

[6] A.C.Paul, "Transport: An Ion Optic Program", Lawrence Berkeley Laboratory, LBL2607, UC-32, TID-4500-R62, Feb. 1975. 
[7] W.B.Colson, J.C Gallardo and P.M.Bosen Phys. Rev. A 34 (1986) 4875.

[8] W.B.Colson, IEEE J. Quantum Elec. QE-17 (1981) 1417.

[9] E.T.Scharlemann and W.M.Fawley, SPIE 642 (1086) 2. 


\begin{tabular}{|l|l|}
\hline Energy & $20 \mathrm{MV}$ \\
\hline Gurrent & $2 \mathrm{kA}$ \\
\hline Radiatlon wavelenglh & $21 \mathrm{\mu m}$ \\
\hline Undulutor period & $2.73 \mathrm{~cm}$ \\
\hline Number of periods & 75 \\
\hline Busm radlus & 1.188 \\
\hline Emlitance (edgo) & $0.4 \mathrm{~cm}$ \\
\hline Raylolgh range & $3400 \mathrm{mrad}-\mathrm{mm}$ \\
\hline
\end{tabular}

Tabłe 1. Conflguratlon paramolers 


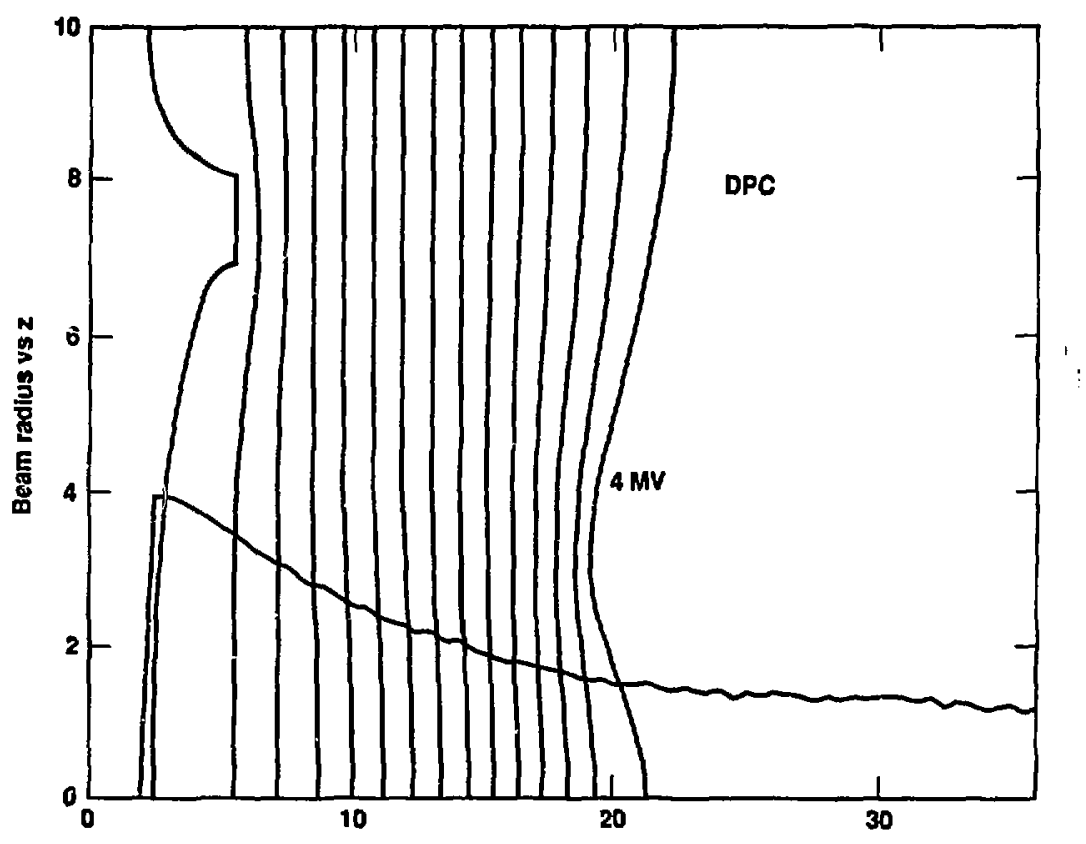

Fig. 1. DPC generated potential contours and beam edge radius 

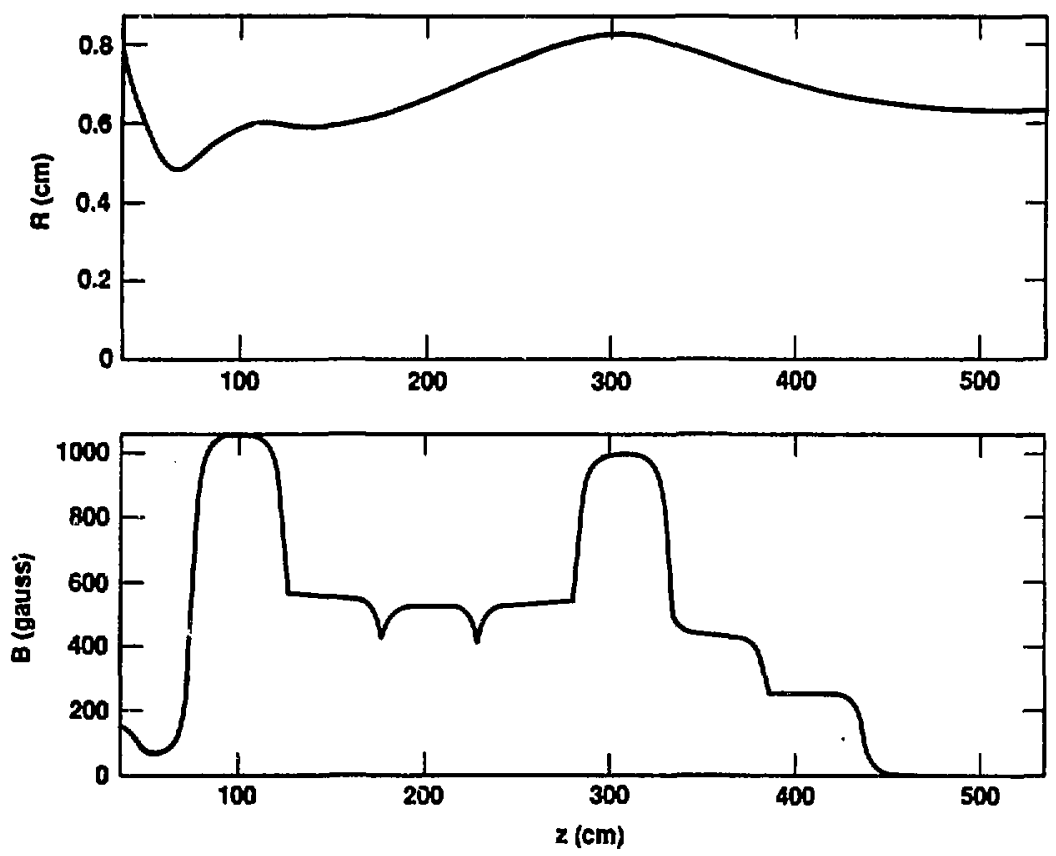

Fig. 2. ST transported rms radius and axis magnetic field 


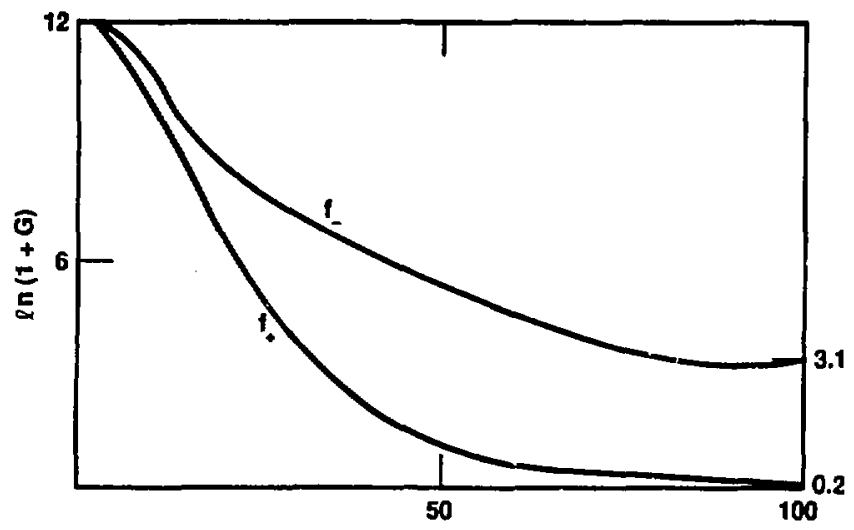

Fig. 3. FEL integral equation gain versus width $\sigma$ 

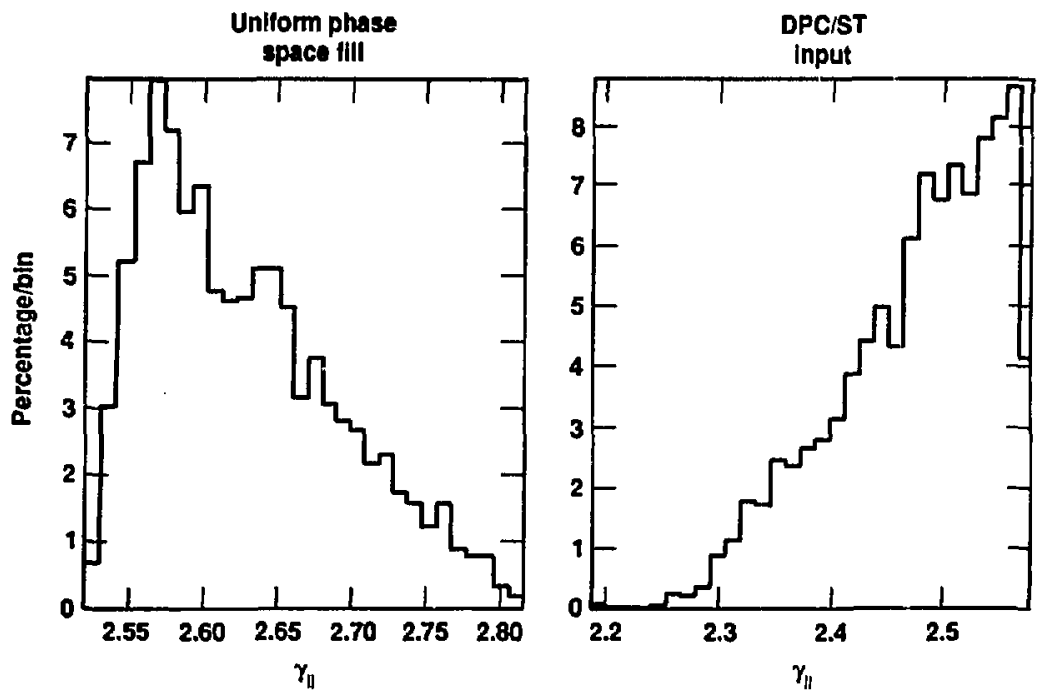

Fig. 4. FRED, parallel energy histograms 


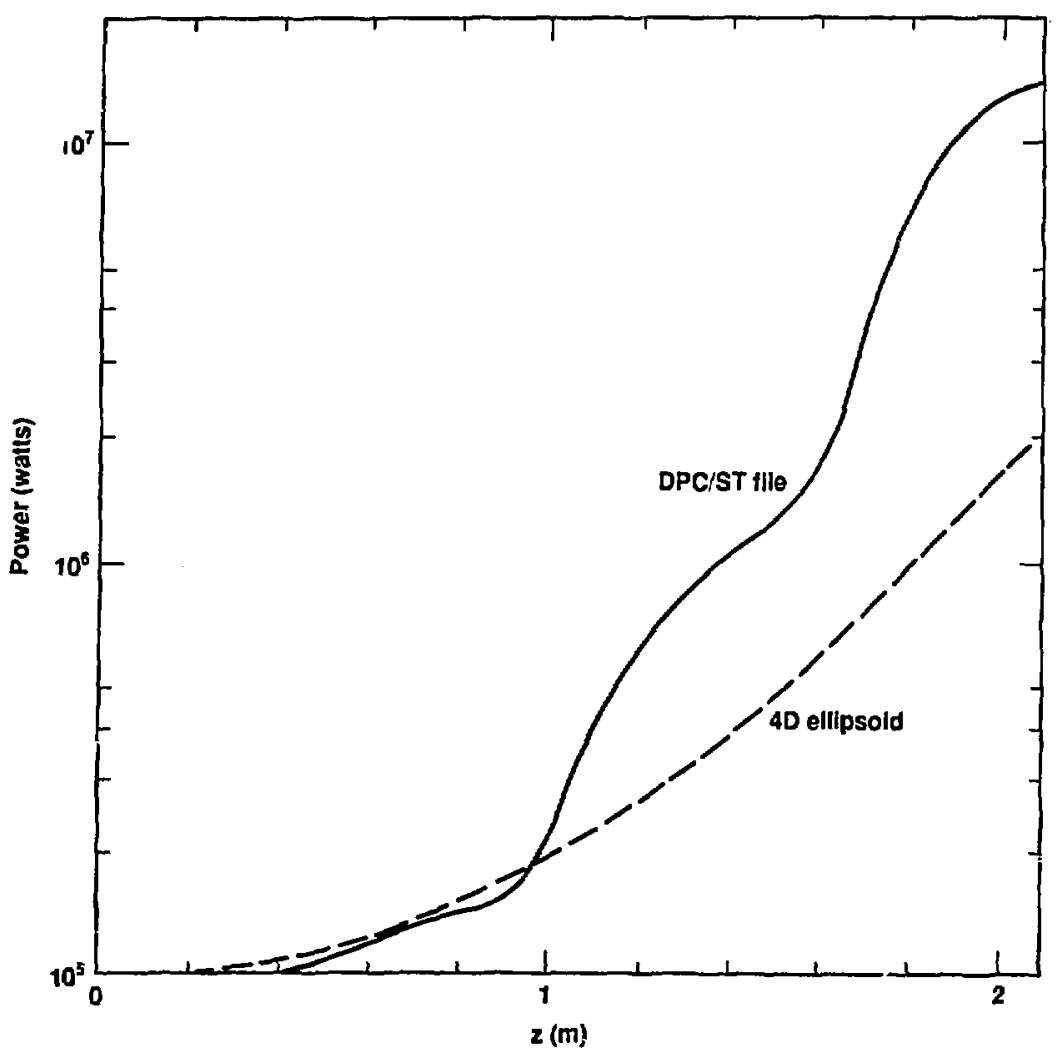

Fig. 5. FRED, power versus distance 